\title{
Routine Microscopy in Quantum Dot Industry
}

\author{
Xiao Feng Zhang ${ }^{1 *}$ \\ 1. Nanosys Inc., 233 S. Hillview Drive, Milpitas, CA 95035 \\ * Corresponding author: xzhang@nanosysinc.com
}

The phrase "quantum dots" is often referred to as semiconductor nanoparticles that emit fluorescence light under excitation by, for example, photon or electron. The diameter of quantum dots ranges from 3 to $10 \mathrm{~nm}$. Nanosys Inc. in Silicon Valley, California is leading the development of quantum dot-based photoluminescence and electroluminescence technologies and products for displays used on tablets, TVs, and monitors that are brighter and more colorful than ever before while consuming less power [1]. Fig. 1 shows a fluorescence micrograph of photoresist patterning of fine features containing quantum dots. While Nanosys is able to supply various quantum dot products for more than 6 million TVs per year, the materials characterization is the back bone of all of the activities in R\&D, product design and engineering, and quality control. In Nanosys, the materials characterization lab performs chemical analysis and microscopy imaging using a line of chemical analytical instruments and optical and electron microscopes. Structure/phase, shape/size/dimension, defects/failure mechanisms, and organic/inorganic constituents are the daily targets. Some typical microscopy works are presented here to demonstrate real-world problem solving in the nanomaterials industry.

Fig. 2(a) shows a typical microscopy work in the nanomaterials industry. Because of the nanoscale size, quantum dots can only be effectively seen using transmission electron microscopy (TEM). Nanoparticles are dispersed on an ultrathin carbon film and imaged with TEM to obtain information about morphology, size and size distribution, phase, and composition. In some products, quantum dots may be embedded in polymers to make quantum dot-enhanced film, which is a key part for the so-called quantum dot TV, or quantum LED can be made to eventually replace organic LED. Fig. 2(b) reveals a middle stage of an electroluminescence device design, in which a monolayer of quantum dot was deposited on a substrate. Because thick substrate is involved, high resolution scanning electron microscopy (SEM) is more appropriate than TEM to show morphology, dimension, dispersion uniformity, area density, and defects of the deposited quantum dot layer. Atomic force microscope (AFM) is sometimes used to determine the surface roughness at a nanometer-scale.

Fig. 3 gives another example of typical daily microscopy work in Nanosys. Quantum dot-containing films are inspected using fluorescence microscope and SEM to see if defects exist and the nature of defects if there is any. In Fig. 3(a), a defect in a green quantum dot-containing film shows a dark contrast in the fluorescence micrograph. The defect is actually a hole formed during spin coating because of a precipitate composed of organics and quantum dots as shown by the SEM image in Fig. 3(b) and associated energy-dispersive X-ray spectroscopy analysis (not shown here).

In conclusion, microscopy characterization is crucial in quantum dot/nanomaterials industry for its unique capability to reveal and characterize nanoscale objects and features. TEM, SEM, AFM, and fluorescence microscope are particularly popular in this industry.

\section{References:}

[1] https://www.nanosysinc.com/ 


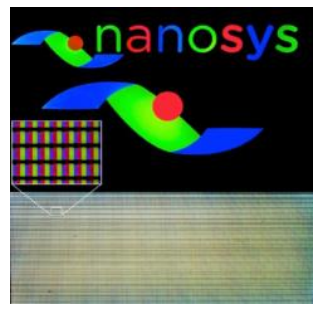

Figure 1. Fluorescence micrograph of a photoresist patterning of fine features containing quantum dots (not visible in the image). The red and green color lights are emitted from quantum dots under blue light illumination.

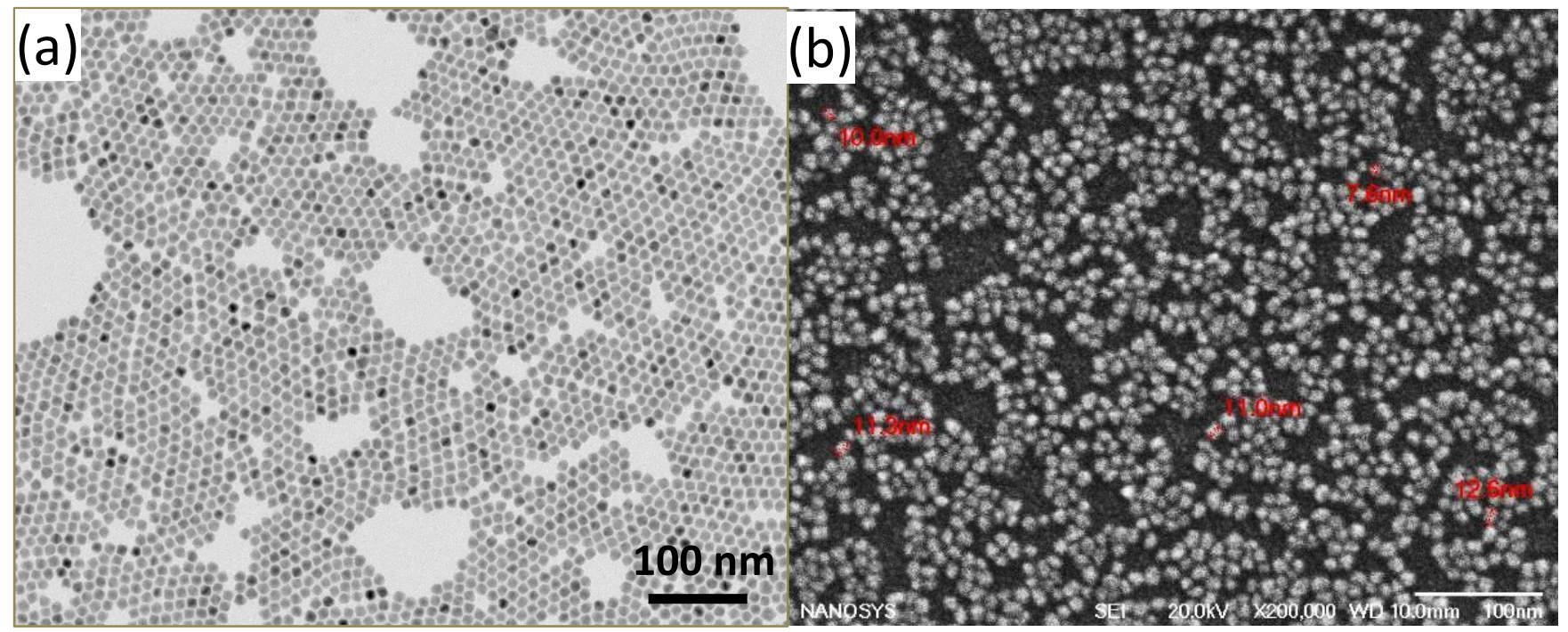

Figure 2. (a) TEM image of quantum dots dispersed on an ultrathin carbon film. (b) High resolution SEM plane-view image showing how dense a monolayer of quantum dot deposited on a substrate. Quantum dot shows bright contrast and substrate is in dark contrast.

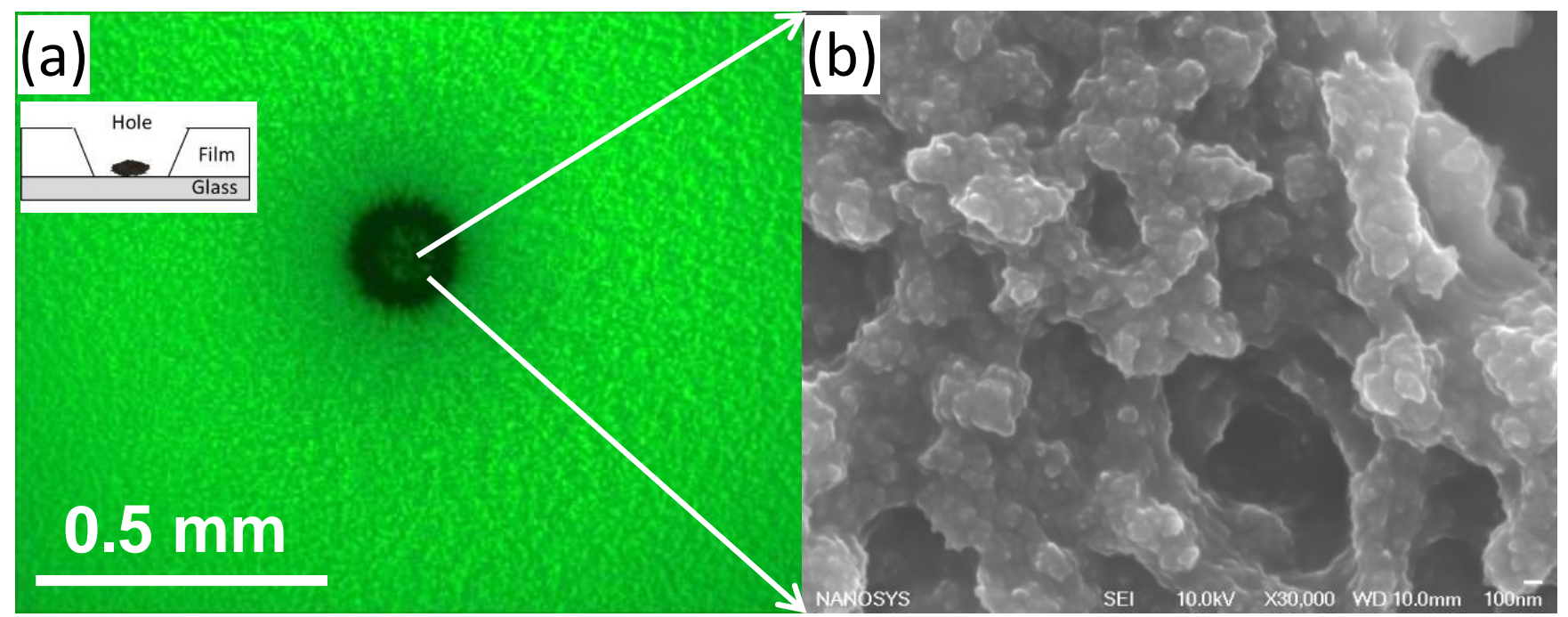

Figure 3. (a) Florescence plane-view micrograph showing a defect in a film made from green quantum dot embedded in epoxy. The defect is a hole (black) throughout the film thickness and filled with impurities. The inset schematically illustrates the geometry from a cross-sectional view direction. (b) SEM image of a zoomed in area on the impurities. The material is gel-like organic mixed with quantum dots. 